\title{
Effect of Dextran Gel on Preparation of Nano-liposomes Loaded with Ginkgolide
}

\author{
Yuan Tong, Yan Chen, ${ }^{*}$ Jian Pan, ${ }^{\dagger}$ Li Huang, and Ruijun Wang \\ School of Life Science, Anhui University, Hefei 230039, P. R. China. *E-mail: chenyan91030@yahoo.com \\ ${ }^{\dagger}$ Engineering Research Center of Bio-Process, Hefei University of Technology, Ministry of Education, \\ Hefei 230009, P. R. China \\ Received March 23, 2010, Accepted July 22, 2010
}

\begin{abstract}
The objective of this paper was to investigate the effect of dextran gel on preparation of nano-liposomes loaded with ginkgolide. During preparation, Sephadex G75, G50 and G25 were added in the aqueous phase respectively. From the experiment, nano-liposomes prepared by dextran gels were found spherical and smooth. The result indicated that aperture of dextran gels were narrower, particle size of nano-liposomes was smaller $(207.13 \sim 89.16 \mathrm{~nm})$ and zeta potential was greater $(-36.2 \sim-29.5 \mathrm{mV})$ in more negative. The study also revealed that differences of the entrapment efficiency and drug loading among the three types of nano-liposomes were not significant. In vitro drug release test demonstrated that nano-liposomes had a better controlled release. To conclude, by using dextran gel in the preparation of nano-liposome loaded with ginkgolide, the particle size could be effectively controlled and the drug stability could be improved.
\end{abstract}

Key Words: Ginkgolide A, Nano-liposomes, Dextran gel, Particle size

\section{Introduction}

Ginkgolide was recognized as platelet-activating factor antagonist which was the main efficacy component from Ginkgo biloba extract and its preparations. Ginkgo biloba terpene lactones $\mathrm{A}$ and $\mathrm{B}$ had neuroprotective effect by inhibiting the protein kinase $\mathrm{C}(\mathrm{PKC})$, so it could treat neuropathy, in particular, demyelization neuropathy, spinal cord disease, and cerebral edema. ${ }^{1,2}$ In addition, the cardiovascular system diseases, acute cerebral infarction, simple diabetic retinopathy could be treated by ginkgolide as the role of effective regulator on arteries, veins and capillaries. ${ }^{3}$ However, because ginkgolide was poorly water-soluble, short biological half-life, its applications in the medicine were seriously restricted. ${ }^{4,5}$

For the purpose of improving the pharmacological properties of ginkgolide, nano-liposome was used as drug carriers, because nano-liposomes were easily biodegradable and biocompatible with lipid-membrane structure. It had the advantages of primarily consisting of protection of biological activity of drugs, improving stability, extending the half-life, controlling drug release, improving efficacy, etc. ${ }^{6}$ At the same time, owing to their small size (about $100 \mathrm{~nm}$ ), nano-liposomes could not only pass through tissue space and be absorbed by cells, pass through the body's smallest capillaries, but also go through the bloodbrain barrier. ${ }^{7-9}$ In recent years, nanoparticles have received considerable attention because of the versatility of polymer matrices, and were allowed for tailoring the nanoparticle properties to meet the specific need. ${ }^{10-12}$ Consequently, it was widely used as carrier in various drug delivery systems. ${ }^{13}$

In vivo, the particle size of nano-liposomes was one of the most important factors considered. ${ }^{14}$ It was shown that the particle size was smaller, the radius of curvature in its circulation would be shorter and the effect of protein absorption would be less. Therefore, half-life of drug could be extended by reducing the particle size, and nano-liposomes had the ability to evade the interception by sinusoidal vessel of liver and spleen. The

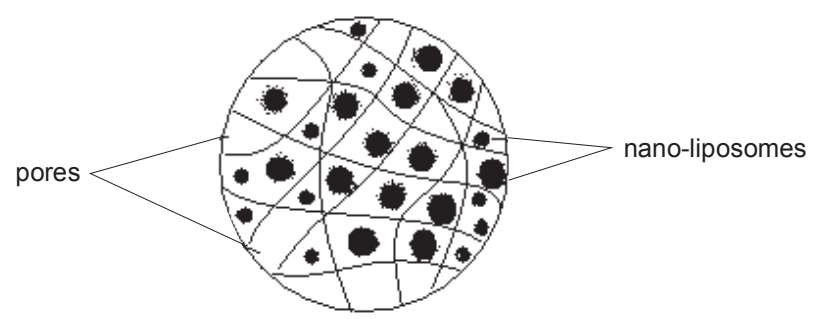

Figure 1. Schematic diagram of dextran gel bead.

current methods of controlling or reducing the particle size during preparation were usually to change the power of ultrasound, stirring speed, temperature, lipid ratio, etc. ${ }^{15}$

The main difference between the work presented here and other optimized works was the adoption of dextran gel-assisted liposome formation method. Dextran gel (commerce name: Sephadex) was commonly used in size exclusion chromatography (SEC) which worked by trapping smaller molecules in the pores of a particle. Dextran gel in this report was not used to separate different size of nano-liposomes, but to assist in limiting the particle size of nano-liposomes inside the pores of the dextran gel beads during the preparation of liposome. The pores in dextran gel bead would support some constraint spaces and when the materials entered the pores, the nano-liposomes with small particle size would be formed (Figure 1). Three kinds of dextran gels (Sephadex G25, G50 and G75) with varying size of pores were selected to state the effect of dextran gel on particle sizes in the process of making liposome.

\section{Experimental Section}

Materials. Sephadex G25 Medium, G50 Medium and G75 Medium were provided by Pharmacia $(50 \sim 300-\mu \mathrm{m}$ beads, Sweden). Ginkgolide A was obtained from Hefei University of Technology (Purity $>95 \%$ ). Standard preparation of ginkgolide A 
was purchased from national institute for the control of pharmaceutical and biological products (China). Phospholipid from egg (egg-PC) was from Sigma (America), cholesterol and phosphatidyl ethanolamine were from Sinopharm Chemical Reagent Co., Ltd (China). Tween- 80 was obtained from Sigma. Organic solvents were of HPLC grade and water was purified by the Milli-Q system.

Pre-treatment of dextran gel. The dry dextran gel would be swollen in water in advance. $6.5 \mathrm{~g}$ Sephadex G75, $10 \mathrm{~g} \mathrm{G} 50$ and $20 \mathrm{~g} \mathrm{G} 25$ were added into $50 \mathrm{~mL}$ distilled water respectively. Sephadex G75 would be boiled for 3 hours, and the boiling time for G50 and G25 would be 1 hour. After the supernatant and fine particles had been removed, the treated gels were made ready for the next step.

Preparation of nano-liposomes. Nano-liposomes were made by the method of ether injection. All lipids were dissolved in $20 \mathrm{~mL}$ of ether then the organic phase was obtained by adding in methanol solution of $100 \mathrm{mg}$ ginkgolide A. $0.3 \mathrm{~mL}$ of Tween80 and $5 \mathrm{~mL}$ of the treated gels were added in the $30 \mathrm{~mL} \mathrm{H}_{2} \mathrm{O}$ as the aqueous phase, $750 \mathrm{rpm}$ stirring at $74{ }^{\circ} \mathrm{C}$. Organic phase was injected into the aqueous solution, which was magnetically stirred and maintained at $74{ }^{\circ} \mathrm{C}$ to evaporate the ether. When the liquid had been evaporated to $10 \mathrm{~mL}, 20 \mathrm{~mL}$ ice water was added in, continuously stirred for $10 \mathrm{~min}$ at the ambient temperature. In order to extract nano-liposomes from dextran gels, suspension was passed through a $0.45 \mu \mathrm{m}$ micron filter. The control and the three kinds of nano-liposomes would be collected: the control was made without dextran gel, sample 1 (S1), sample 2 (S2) and sample 3 (S3) were prepared by Sephadex G75, G50 and G25, respectively.

Transmission electron microscopy (TEM). The nano-liposomes were stained with a $2 \%(\mathrm{w} / \mathrm{v})$ solution of phosphotungstic acid, respectively. Then, $5 \mu \mathrm{L}$ of each sample was placed on a copper micro-grid (230 mesh), the grid was dried and transmission electron microscopic (TEM) observation (JEM-100SX, Japan) was performed using the dried micro-grid and $100 \mathrm{kV}$ acceleration voltage. Images were recorded directly in electron microscopic films. Films were then developed and analyzed.

Particle size and zeta potential measurement. The mean particle size and zeta potential of the systems were measured by Zeta-sizer 3000HS (Malvern, UK). The measurements were carried out at a scattering angle of $90^{\circ}$ and the ambient temperature. 1:10 dilution of the formulations was prepared in doubledistilled water before the measurement.

Entrapment efficiency (EE) and drug loading (DL). For the quantitative determination of ginkgolide A, a method of highspeed refrigerated centrifuge was used. Ginkgolide A loaded into the nano-liposomes was determined as follows: $1.5 \mathrm{~mL}$ of $1 \mathrm{M}$ hydrochloric acid was added in $1 \mathrm{~mL}$ of freshly prepared nano-liposomes suspension and the mixture was centrifuged at $15,000 \times g$ for $120 \mathrm{~min}$ at $4{ }^{\circ} \mathrm{C}$ using a Sigma $3 \mathrm{~K} 15$ centrifuge (Germany). The total drug in supernatant after centrifugation was determined by the current high performance liquid chromatography (HPLC) method. ${ }^{18,19}$ The HPLC system which were equipped with an evaporative light-scattering detector (ELSD, Waters 2420) consisted of an isocratic pump (Waters 515 pumps, Waters Corporation), a $\mathrm{C}_{18}$ analytical column (4.6 mm $\times 250$ $\mathrm{mm}, 5 \mu \mathrm{m}$ particle size) (Restek pinnacle), and a guard column
$(5 \mathrm{~cm})$ (Agilent) used to protect the analytical column. Mobile phase was filtered through $0.2 \mu \mathrm{m}$ nylon 66 filters which was freshly prepared each day and pumped at $1 \mathrm{~mL} / \mathrm{min}$. Mobile phase was prepared by mixing water, methanol and tetrahydrofuran at a ratio of 70:25:10 v/v/v. ELSD was used as the detector with nebulizing nitrogen pressure of $25 \mathrm{psi}$ and the drift tube temperature of $55^{\circ} \mathrm{C}$. The determination was performed at ambient temperature. The injection volume was $10 \mu \mathrm{L} .^{20}$

The EE of ginkgolide A in nano-liposomes was determined, as to the ratio between actual and theoretical loading, using the following equation: ${ }^{21}$

$$
\mathrm{EE}(\%)=\frac{\text { amount of drug in liposome }}{\text { amount of drug added }} \times 100
$$

Drug loading capacity (DL) was calculated as drug analyzed in the nanoparticles versus the total amount of the drug and the excipients added during preparation, according to the following equation:

$$
\begin{aligned}
& \mathrm{DL}(\%) \\
& =\frac{\text { amount of drug loaded in liposome }}{\text { amount of drug added }+ \text { amount of excipients added }} \times 100
\end{aligned}
$$

Stability. Three kinds of nano-liposomes suspension prepared by dextran gels were sealed and stored at $4{ }^{\circ} \mathrm{C}$. The appearances of morphological characteristics of nano-liposomes were observed and the particle sizes were measured continuously in $30 \mathrm{~d}$.

In vitro drug release. In vitro release study was performed on suspension of nanoparticles within $24 \mathrm{~h}$ of preparation. ${ }^{22,23} 2 \mathrm{~mL}$ of dispersion and $3 \mathrm{~mL}$ phosphate buffer solution ( $\mathrm{pH} 7.4$ ) were transferred to a dialysis tube (molecular weight cutoff 3500 ), and the sealed tube was introduced into a vial containing $50 \mathrm{~mL}$ of phosphate buffer solution. Bottles were placed in a water bath under mild agitation $(50 \mathrm{rpm})$ at $37 \pm 1{ }^{\circ} \mathrm{C} .{ }^{24}$ At predetermined time intervals from 0 to $72 \mathrm{~h}, 1 \mathrm{~mL}$ sample of the medium was taken out and replaced with the same amount of fresh medium. The drug amount in the release medium was determined by HPLC, respectively. ${ }^{25}$ At intervals of 5 minutes, the release of ginkgolide A was determined with the same method as before.

Statistical analysis. One-way analysis of variance (ANOVA) test was performed on the data to assess the impact of the formulation variables on the results. $P$ values of $<0.05$ or 0.01 were considered significant. All calculations were performed using the Statistical Package for the Social Sciences $\left(\operatorname{SPSS}^{\circledR} 17.0\right)$ software.

\section{Results and Discussion}

Transmission electron microscope (TEM). The control and three samples were pallide-flavens and non-stratified. Additionally, there were not any precipitation and agglomeration in observation. To obtain more information about the particle size and morphology, a TEM analysis was also performed. Figure 2 showed images of nano-liposomes which were prepared by blank, Sehpadex G75, G50 and G25, respectively. According to 

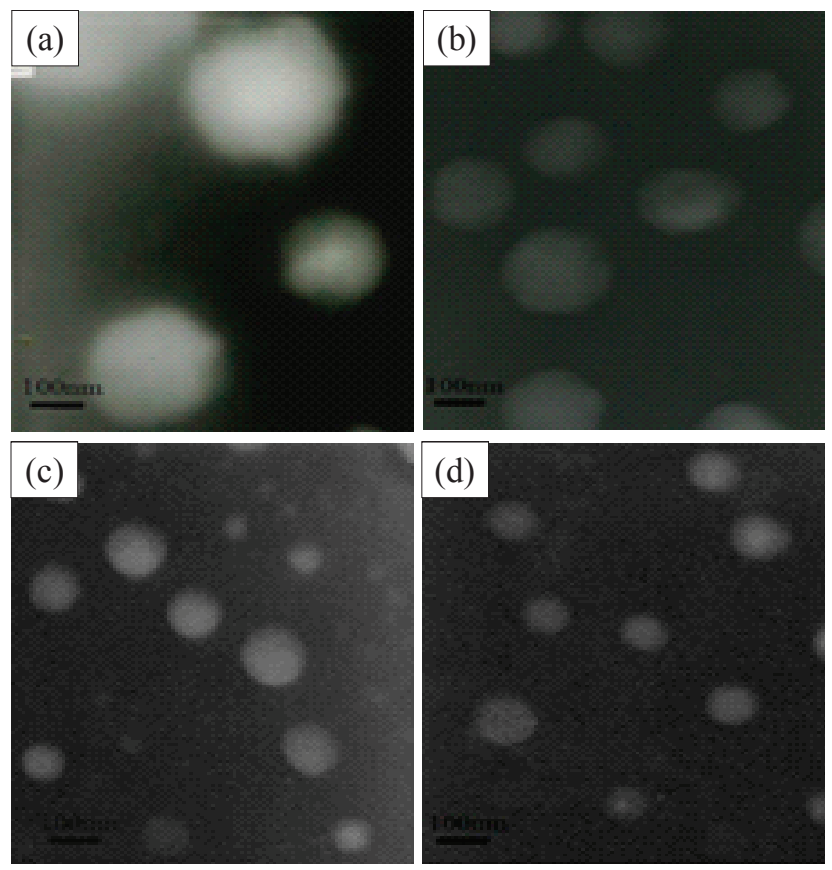

Figure 2. Transmission electron microscope (TEM) micrographs. (A) The control (×50 000), (B) S1 (×50 000), (C) S2 (×50 000), D: S3 $(\times 50000)$.

the TEM micrographs, the particle size distribution of the S1, S2 and S3 showed better distribution than the control. Furthermore, the liposomes which were prepared by Sephadex G25 and G50 appeared better spherical shape and monodispersed size distribution.

Particle size and zeta potential. In the case of dextran gel, the average particle size was between 89.16 and $207.13 \mathrm{~nm}$ which were smaller than the control, and the zeta potential was between -29.5 and $-36.2 \mathrm{mV}$, as shown in Table 1. With the diminution of the apertures in the dextran gels, the particle size and zeta potentials were both reduced. Compared with others, the $\mathrm{S} 3$ exhibited the smallest particle size $(P<0.01)$ in the range of 45.42 to $143.51 \mathrm{~nm}$, and much narrower and more even size distribution for $80.5 \%$ of the total nano-liposomes were less than $100 \mathrm{~nm}$. Additionally, the absolute value of zeta potential was $36.2 \mathrm{mV}$, which was significantly high $(P<0.01)$ among all samples. Therefore, the nano-liposomes with relative high stability and good dispersion quality were produced by this method.

Entrapment efficiency (EE) and drug loading (DL). Calibration curve was constructed by analyzing a serial of ginkgolide A at the concentration from 0.2 to $1.0 \mathrm{mg} / \mathrm{mL}$. Preparation and supernatant of the samples were carried out as described under assay procedure. Peak area $(y)$ of the samples was measured and plotted against the concentration $(x)$ of each ginkgolide A. The regression equations of the calibration curve was $\lg y=$ $1.6686 \lg x+7.4599(\gamma=0.9993)$.

Analytical recovery studies were performed by spiking samples with known amounts of ginkgolide A standard in methanol from 0.4 to $0.8 \mathrm{mg} / \mathrm{mL}$. Mean recoveries rates were determined based on triplicate analyses at each level. As the result, there was a high recovery rate of $(100.5 \pm 1.15) \%$.

For peak identification, the retention times of pure substances
Table 1. The characterization of nano-liposome by particle size and zeta potential

\begin{tabular}{ccl}
\hline Nano-liposome & Particle size $(\mathrm{nm})$ & Zeta potential $(\mathrm{mV})$ \\
\hline S 1 & $207.13 \pm 18.06(\mathrm{P}<0.05)$ & $-29.5 \pm 3.4(\mathrm{P}<0.05)$ \\
S 2 & $162.45 \pm 14.17(\mathrm{P}<0.01)$ & $-33.6 \pm 2.2(\mathrm{P}<0.01)$ \\
S 3 & $89.16 \pm 9.20(\mathrm{P}<0.01)$ & $-36.2 \pm 2.8(\mathrm{P}<0.01)$ \\
The control & $239.00 \pm 24.34$ & $-23.7 \pm 4.1$
\end{tabular}

Each value represents the mean $\pm \mathrm{SD}(n=5)$

Table 2. Recovery studies using high-performance liquid chromatography (HPLC)

\begin{tabular}{ccrc}
\hline $\begin{array}{c}\text { Sample } \\
\text { concentration } \\
(\mathrm{mg} / \mathrm{mL})\end{array}$ & $\begin{array}{c}\text { Determination } \\
\text { of concentration } \\
(\mathrm{mg} / \mathrm{mL})\end{array}$ & Recovery & RSD $(\%)$ \\
\hline 0.4 & $0.402 \pm 0.02$ & $100.5 \%$ & \\
0.6 & $0.596 \pm 0.03$ & $99.3 \%$ & $0.54 \%$ \\
0.8 & $0.813 \pm 0.05$ & $101.6 \%$ & \\
\hline
\end{tabular}

Each value represents the mean $\pm \mathrm{SD}(n=3)$

had been used. It could be seen from chromatograms Figure 3 (A and B) that there was no interference to determine the main drug for the peak of blank liposome only in $4 \mathrm{~min}$, but the peak of ginkgolide A occurred at about 11 min (Figure 3, C).

The prerequisite to obtain a sufficient loading capacity was a sufficiently high solubility of the drug in the lipid melt. Relative higher drug EE was one of the major advantages of liposomes. The EE and DL of the three samples prepared by dextran gels and the control were shown in Table 3. The differences of EE and DL between three kinds of nano-liposomes prepared by dextran gels and the control were statistically significant $(P<$ 0.05 or 0.01$)$, but there was no significant difference $(P>0.05)$ among the three samples. The minimum loading of $\mathrm{S} 3$ reached $15.20 \%$, suggesting an entrapment efficiency of $89.85 \%$. As was evident in Figure 4, the particle sizes were different with the three dextran gels, although the sizes were diminished, the changes of EE were not so intense. On the basis of these physical and chemical properties, it seemed that nano-liposomes loaded with ginkgolide A prepared by Sephadex G25 would be better than the others.

Stability. As shown in TEM image, the particle size did not change significantly after storage for $30 d$ at $4{ }^{\circ} \mathrm{C}$. The particle size increased slightly $(P>0.05)$ compared to the freshly prepared samples, as storage time extended. The results of the stability study revealed that the prepared samples were stable for more than $30 d$ at $4{ }^{\circ} \mathrm{C}$ and reflected good long-term stability of the nano-liposomes.

In vitro drug release. A key issue investigated in this study was the feasibility of using nano-liposomes to deliver ginkgolide A. Results of the in vitro study on ginkgolide A released from the nano-liposomes were shown in Figure 5. The percentages of drug released from each carrier were plotted as a function of time. Since drug release in the initial stage would be entirely significant if the drug were distributed over the particle surface, ${ }^{26}$ the ginkgolide A appeared to be released from nano-lipo- 

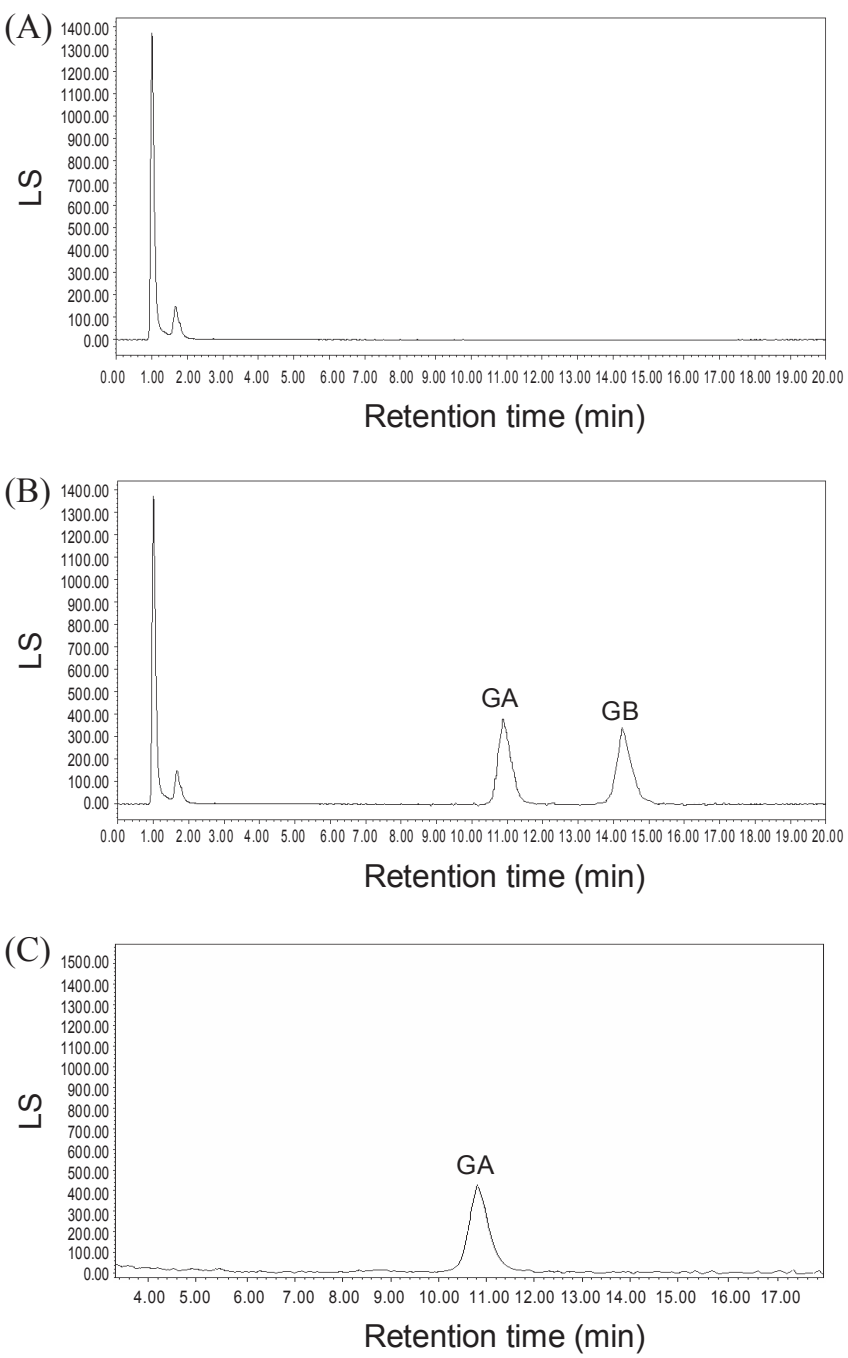

Figure 3. HPLC chromatograms. (A) Blank liposome, (B) Pure drug of ginkgolide A, B standard mixed solution with blank liposome, (C) Ginkgolide A in supernatant of nano-liposome.

Table 3. Entrapment efficiency (EE) and drug loading (DL)

\begin{tabular}{ccc}
\hline Nano-liposomes & $\begin{array}{c}\text { Entrapment efficiency } \\
(\%)\end{array}$ & $\begin{array}{c}\text { Drug loading } \\
(\%)\end{array}$ \\
\hline S1 & $91.19 \pm 1.21(\mathrm{P}<0.05)$ & $16.02 \pm 0.43(\mathrm{P}<0.01)$ \\
S2 & $90.33 \pm 0.79(\mathrm{P}<0.05)$ & $15.73 \pm 0.44(\mathrm{P}<0.01)$ \\
S3 & $89.85 \pm 0.68(\mathrm{P}<0.05)$ & $15.20 \pm 0.32(\mathrm{P}<0.01)$ \\
The control & $93.02 \pm 1.89$ & $17.65 \pm 0.63$
\end{tabular}

Each value represents the mean $\pm \mathrm{SD}(n=5)$

somes in a biphasic way, which characterized by an initial release or rapid release period followed by a step of slower release. ${ }^{27}$ The burst effect was observed in $12 \mathrm{~h}$, in which $41.63 \%$ to $56.39 \%$ of the initial drug was released from nano-liposomes, and the cumulative release rates of S2, S3 and S1 were in an increasing order. After this initial effect, ginkgoide A was released in a continuous way for up to $72 \mathrm{~h}$, reaching percentage of cumulative release close to $70.86 \%$. However, for the pure ginkgolide A solution, about $94.57 \%$ of the drug was released in $2 \mathrm{~h}$, and the

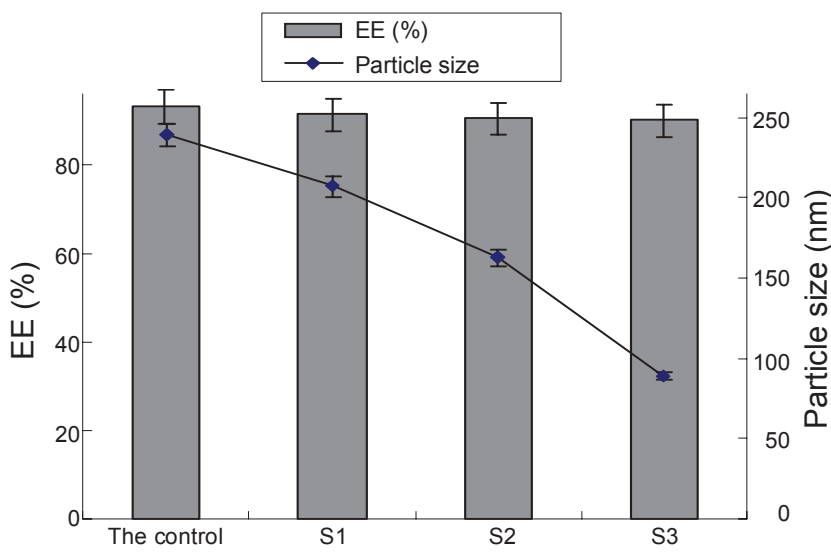

Figure 4. Effect of dextran gels on the average size and EE of the nano-liposomes. Each value represents the mean $\pm \mathrm{SD}(n=5)$.

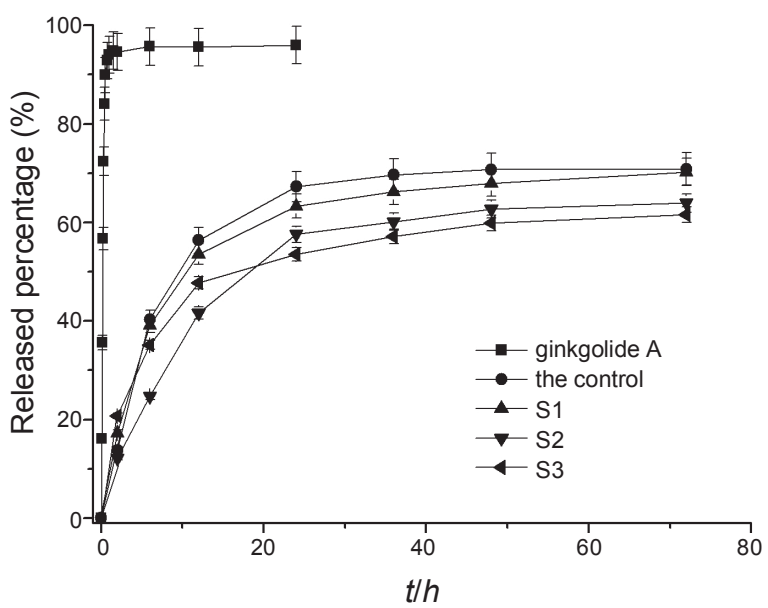

Figure 5. In vitro release curve of ginkgolide A. Each value represents the mean $\pm \mathrm{SD}(n=4)$.

release amount reached the maximum after $6 \mathrm{~h}$. This suggested that nano-liposomes prepared by dextran gels not only possessed a small particle size, but had a good effect on delayed release of poor water-soluble ginkgolide A as well.

\section{Conclusion}

The amount of drug to be incorporated into the delivery system is dependent on the physicochemical properties of drug and the preparation process. ${ }^{28}$ The significant finding of this study was that tiny nano-liposomes with high ginkgolide A content and good stability were successfully developed by the means of dextran gels. In this report, dextran gels were added in the aqueous phase to prepare nano-liposomes loaded with ginkgolide A and the results showed that the particle size of nanoliposomes could be controlled with the pores of dextran gel in the process of making liposome. Morphology and stability of nano-liposomes were demonstrated better after using dextran gels and when the apertures of dextran gels were narrower, the nano-liposomes were also smaller.

This report offered a new practical method to make nanoliposomes loaded with ginkgolide and further studies of how 
to prepare tinier particle size using other types of molecular gels would be demanded.

Acknowledgments. This study was supported by Foundation for Key Research Program of Education Department of Anhui Province, China (KJ2009A172) and Natural Science Foundation of Anhui Province, China (070413143).

\section{References}

1. Ahlemeyer, B.; Krieglstein, J. J. Cell. Mol. Life Sci. 2003, 60, 1779.

2. Sakakibara, H.; Izawa, Y.; Nakajima, J.; Seo, S.; Tamaki, T.; Kawai, Y.; Terao, J. Functional Food and Health; University Press: Oxford, U. K., 2008; p 429.

3. Chen, Z. Y.; Peng, C.; Jiao, R.; Wong, Y. M.; Yang, N.; Huang, Y. J. Agric. Food Chem. 2009, 57, 4485.

4. Mauri, P.; Simonetti, P.; Gardana, C.; Minoggio, M.; Morazzoni, P.; Bombardelli, E.; Pietta, P. J. Rapid commun Mass Spectrom. 2001, 15, 929

5. Lv, H.; Wang, G. J.; Li, H.; Huang, M. W.; Xie, H. T.; Huang, C. R.; Sun, J. G.; Lv, T. J. Pharm. Biom. 2006, 40, 88

6. Müller, R. H.; Maaßen, S.; Weyhers, H.; Specht, F.; Lucks, J. S. Int. J. Pharm. 1996, 138, 85.

7. Dimitrov, D. S. J. Colloids Surf. A 2006, 282, 8.

8. Liu, G.; Men, P.; Harris, P. L. R.; Rolston, R. K.; Perry, G.; Smith, M. A. J. Neurosci Lett. 2006, 406, 189.

9. Schnyder, A.; Huwyler, J. J. Neurotherapeutics 2005, 2, 99.

10. Kim, K.; Lee, M.; Park, H.; Kim, J. H.; Kim, S. W.; Chung, H.; Choi, K.; Kim, I. S.; Seong, B. L.; Kwon, I. C. J. Am. Chem. Soc. 2006, $128,3490$.

11. Moura, M. R.; Aouada, F. A.; Mattoso, L. H. C. J. Colloid Interface Sci. 2008, 321, 477.
12. Farah, A. A.; Alvarez-Puebla, R. A.; Fenniri, H. J. Colloid Interface Sci. 2008, 319, 572.

13. Müller, R. H.; Mäder, K.; Gohla, S. Euro. J. Pharm. Biopharm. 2000, 50, 161.

14. Abra, R. M.; Hunt, C. A. J. Biochimica et Biophysica Acta (BBA)Lipids and Lipid Metabolism 1981, 666, 493.

15. An, S. Y.; Bui, M. N.; Nam, Y. J.; Han, K. N. J. Colloid Interface Sci. 2009, 331, 98 .

16. Mathai, J. C.; Sitaraman, V. J. Biochemical Education 1987, 15, 147

17. Pham, H. L.; Shaw, P. N.; Davies, N. M. J. Int. Pharm. 2006, 310, 196

18. Dubber, M.-J.; Kanfer, I. J. Pharm. Biom. 2006, 41, 135.

19. Mesbah, M. K.; Khalifa, S. I.; El-Gindy, A.; Tawfik, K. A. J. IL Farmaco 2005, 60, 583.

20. Mesbah, M. K.; Khalifa, S. I.; El-Gindy, A.; Tawfik, K. A. J. IL Farmaco 2005, 60, 583

21. Subedi, R. K.; Kang, K. W.; Choi, H. K. Euro. J. Pharm. 2009, 37, 508

22. Schwab, M.; Kessler, B.; Wolf, E.; Jordan, G.; Mohl, S.; Winter, G. J. Euro. Pharm. Biopharm. 2008, 70, 690.

23. Larsen, S. W.; Frost, A. B.; Østergaard, J.; Marcher, H.; Larsen, C. J. Euro. Pharm. Sci. 2008, 34, 37.

24. Mourtas, S.; Fotopoulou, S.; Duraj, S.; Sfika, V.; Tsakiroglou, C.; Antimisiaris, S. G. J. Colloids and Surfaces B: Biointerfaces 2007, 55,212

25. Liu, K. S.; Tzeng, J. I.; Chen, Y. W.; Huang, K. L.; Kuei, C. H.; Wang, J. J. Anesth. Analg. 2006, 102, 1445.

26. Kuo, Y. C.; Chen, H. H. Int. J. Pharm. 2009, 365, 206.

27. Merodio, M.; Arnedo, A.; Renedo, M. J.; Irache, J. M. J. Euro. Pharm. Sci. 2001, 12, 251.

28. Li, Y. C.; Dong, L.; Jia, A.; Chang, X. M.; Xue, H. J. Int. Bio. Macromolecules 2006, 38, 296. 